\title{
Hyperlipidemia, CTCAE
}

National Cancer Institute

\section{Source}

National Cancer Institute. Hyperlipidemia, CT CAE. NCI Thesaurus. Code C143221.

A disorder characterized by laboratory test results that indicate an elevation in the concentration of lipids in blood. 